\title{
Primary Peritoneal High-grade Serous Carcinoma Misinterpreted as Metastatic Breast Carcinoma: A Rare Encounter in Peritoneal Fluid Cytology
}

\author{
SUJIN PARK ${ }^{1}$, EUN YOON CHO ${ }^{1}$, YOUNG LYUN OH ${ }^{1}$, YEON HEE PARK ${ }^{2}$ and HYUN-SOO KIM ${ }^{1}$ \\ ${ }^{1}$ Department of Pathology and Translational Genomics, Samsung Medical Center, \\ Sungkyunkwan University School of Medicine, Seoul, Republic of Korea; \\ ${ }^{2}$ Division of Hematology-Oncology, Department of Medicine, Samsung Medical Center, \\ Sungkyunkwan University School of Medicine, Seoul, Republic of Korea
}

\begin{abstract}
Background/Aim: Peritoneal fluid (PF) cytology is critical for distinguishing high-grade serous carcinoma (HGSC) from metastatic disease in patients with breast carcinoma who present with peritoneal carcinomatosis $(P C)$. Case Report: A 50-year-old woman underwent surgery and adjuvant therapy for pTINO grade $2 / 2$ luminal A breast carcinoma. Sixteen months postoperatively, palliative chemotherapy was administered following a pleural biopsy and diagnosis of metastatic carcinoma. The patient developed PC despite chemotherapy. PF cytology specimens suggested metastatic carcinoma. However, we observed a papillary cellular arrangement during the review of cytology slides. HGSC was confirmed by immunocytochemistry showing positive paired box 8 (PAX8) and Wilms' tumor 1 (WT1) expression and negative GATA-binding protein 3 expression. Conclusion: In patients with breast carcinoma history, an awareness of characteristic cytomorphology of HGSC, including a papillary pattern with positive PAX8 and WT1 immunoreactivity, is essential to prevent the misdiagnosis of such cases and in ensuring accurate treatment and management.
\end{abstract}

Peritoneal fluid cytology is a widely used tool for the detection and diagnosis of disseminated malignant cells. However, peritoneal fluid specimens from patients who were treated for breast carcinoma should be examined cautiously

Correspondence to: Hyun-Soo Kim, Department of Pathology and Translational Genomics, Samsung Medical Center, Sungkyunkwan University School of Medicine, 81, Irwon-ro, Gangnam-gu, Seoul 06351, Republic of Korea. Tel: +82 234101243, Fax: +82 234142831, e-mail: hyun-soo.kim@samsung.com

Key Words: Peritoneum, cytology, high-grade serous carcinoma, metastatic breast carcinoma. to ensure a clear distinction between primary peritoneal highgrade serous carcinoma (HGSC) and peritoneal metastatic breast carcinoma (pMBC) $(1,2)$. In patients with peritoneal carcinomatosis, this diagnostic distinction between HGSC and $\mathrm{pMBC}$ is needed to ensure an appropriate treatment strategy (1).

According to previous studies, patients with primary peritoneal HGSC have a significantly longer survival duration than patients with $\mathrm{pMBC}$ after treatment with cytoreductive surgery $(1,3)$. In other words, the origin of peritoneal malignancy is a pivotal factor in the survival prognosis of the patient. Consequently, peritoneal fluid cytology is among the most critical types of assessment performed upon the detection of peritoneal carcinomatosis in a patient with breast carcinoma (1). We herein present a case of a 50-year-old woman with breast carcinoma who was finally diagnosed with peritoneal HGSC after several misdiagnoses of pMBC based on cytological and surgical specimens. An awareness of this repeated misinterpretation prompted us to initiate a clinicopathological review. In this report, we describe the cytopathological and immunophenotypic characteristics that are useful for the distinction of primary peritoneal HGSC from pMBC.

\section{Case Report}

Case presentation. A 50-year-old Korean woman with a family history of breast carcinoma presented with abnormal findings in her left breast that were detected during regular screening. A subsequent biopsy led to a diagnosis of invasive ductal carcinoma of the breast. Magnetic resonance imaging (MRI) revealed an irregular spiculated mass with a diameter of $1.6 \mathrm{~cm}$ in the upper outer quadrant of her right breast, with adjacent probable satellite nodules. A 1.1-cm-sized nonmass enhancement was also detected in the upper outer quadrant of left breast. The patient underwent bilateral 
breast-conserving surgery with sentinel lymph node biopsy.

The postoperative pathological examination led to the diagnosis of multiple invasive ductal carcinomas (two rightsided; one left-sided). All lesions were defined as a modified Scarff-Bloom-Richardson grade of 2. The greatest tumor dimension was $1.5 \mathrm{~cm}$. Immunohistochemistry revealed that the tumors were consistent with the luminal A subtype, as indicated by uniform estrogen receptor (ER; intensity score $=3$; proportion score $=5$; total score $=8$ ) and progesterone receptor (PR) positivity (intensity score $=3$; proportion score $=5$; total score $=8)$ and human epidermal growth factor receptor 2 (HER2; score=0) negativity. An extensive intraductal component was present. Although lymphovascular invasion was identified, no lymph node metastasis was observed. Accordingly, the pathological stage of the breast carcinoma was pT1(3)N0. Postoperatively, the patient received chemotherapy with tamoxifen and goserelin and subsequent adjuvant radiotherapy.

Sixteen months after treatment, this patient presented at the Emergency Department with resting dyspnea. Chest computed tomography (CT) revealed right pleural thickening accompanied by a large volume of effusion in the right hemithorax, suggesting a malignancy. A cytological examination of the right-sided pleural fluid revealed numerous malignant cells, leading to a diagnosis of metastatic carcinoma. A pleural biopsy was performed via video-assisted thoracoscopic surgery (VATS), and an analysis of the specimen supported the apparent diagnosis of metastatic carcinoma. Immunostaining revealed that the pleural tumor was positive for ER but negative for PR and HER2. Accordingly, the patient underwent palliative chemotherapy with paclitaxel for pMBC.

After six cycles of palliative chemotherapy, she presented at the Emergency Department with abdominal distension. Abdominopelvic CT revealed diffuse peritoneal carcinomatosis and a large volume of ascites. The initial cytological analysis of the peritoneal fluid, as well as six subsequent analyses, yielded a diagnosis of metastatic carcinoma. At that time, polymerase chain reaction and sequencing analyses of the breast cancer 1 (BRCA1) and BRCA2 genes revealed no pathogenic mutations.

Twenty-seven months after the initial diagnosis of primary breast carcinoma, a seventh sample of peritoneal fluid was submitted for cytological examination. At that time, a gynecological pathologist examined the liquid-based cytology slides and raised a suspicion of primary peritoneal HGSC. Immunocytochemical staining to detect paired box 8 (PAX8), Wilms tumor 1 (WT1), p53, and GATA-binding protein 3 (GATA3) revealed diffuse and strongly positive staining for the former two proteins but negative staining for the latter two. These results were consistent with tuboovarian or peritoneal serous carcinoma. Subsequent immunostaining revealed a complete loss of p53 expression, indicative of HGSC. Finally, a diagnosis of primary peritoneal HGSC was made based on the absence of tuboovarian lesions on radiologic images. At that time, we repeated the $B R C A$ mutational analysis using a multiplex ligation-dependent probe amplification method and detected a heterozygous deletion of exons 1-14 of BRCA1. Based on these results, the chemotherapy regimen was changed to paclitaxel and carboplatin for the treatment of primary peritoneal HGSC.

The patient's serum cancer antigen-125 concentration decreased rapidly following the initiation of chemotherapy (from 302.9 to $44.1 \mathrm{U} / \mathrm{ml}$ ), indicating a reduced tumor burden. Two months after the change in the chemotherapy regimen, chest $\mathrm{CT}$ revealed no change in the extent of pleural thickening despite the disappearance of the left pleural effusion. Abdominopelvic CT revealed that although the retroperitoneal lymph node metastasis had decreased markedly in size, the mild peritoneal thickening and ascites remained unchanged. Persistent left hydronephrosis indicative of ureteral metastasis was also observed. Taken together, these findings suggested that patient had achieved a partial response.

Cytopathological findings. All liquid-based pleural and peritoneal fluid cytology specimens exhibited high cellularity. A low-power microscopic analysis revealed that the tumor cells were arranged in a predominantly papillary pattern (Figure 1A) and had formed variably sized papillary fragments and clusters with or without fibrovascular cores (Figure 1B). In some areas, individual tumor cells containing large, round-to-oval, hyperchromatic nuclei with pleomorphism and conspicuous nucleoli were admixed with mesothelial cells and inflammatory cells (Figure 1C). Mitoses were frequently observed (Figure 1D), and atypical mitotic figures were present.

The peritoneal fluid cytology specimens collected after chemotherapy contained multiple highly abnormal anaplastic nuclei that were more than five times larger than the adjacent tumor cell nuclei (Figure 1E). Most nuclei were surrounded by irregular nuclear membranes, and some displayed chromatin patterns with a smudged and degenerated appearance. Occasionally, the abnormal nuclear shape even mimicked the appearance of a multinucleated giant cell. Cells harboring these abnormal nuclei also possessed intracytoplasmic microvacuoles (Figure 1F), the aggregates of which resulted in a foamy appearance. Some tumor cells also contained large intracytoplasmic vacuoles that resembled perinuclear halos. Cyst-like spaces were observed between the individual tumor cells (Figure 1G).

Immunostaining of cell blocks and liquid-based preparation slides was performed as previously described (4-15). Immunocytochemically, the tumor cells exhibited diffuse and strong nuclear immunoreactivity for PAX8 (Figure 1H) and 

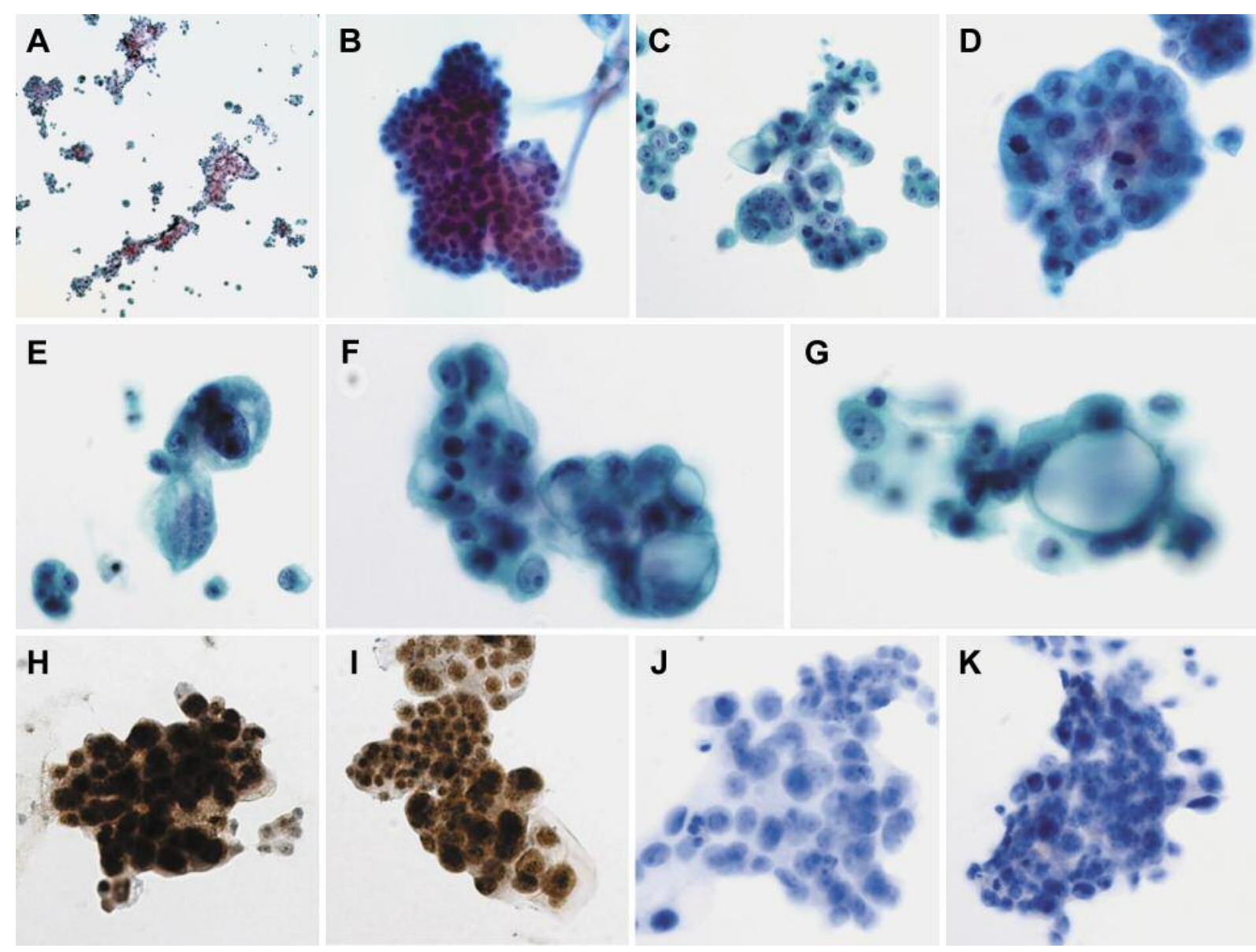

Figure 1. Cytopathological observations of liquid-based preparations of pleural and peritoneal fluid specimens. A: High cellularity with papillary architecture. B: Variably sized papillary fragments and clusters, with or without fibrovascular cores. C: Large, round-to-oval hyperchromatic nuclei with pleomorphism and conspicuous nucleoli. D: Frequent mitoses. E: Chemotherapy-induced atypia, including highly abnormal anaplastic nuclei more than 5 times larger than the adjacent tumor cell nuclei. Chemotherapy-induced atypia included intracytoplasmic microvacuoles $(F)$ and large intracytoplasmic vacuoles $(G)$ that formed perinuclear haloes and cyst-like spaces. Strong, uniform nuclear paired box $8(H)$ and Wilms tumor 1 (I) expression was apparent. J: There was complete absence of p53 expression, indicating TP53 mutation. K: Lack of nuclear GATA-binding protein 3 expression was noted. Liquid-based preparation. A to $G$, Papanicolaou staining; $H$ to $K$, polymer method. Original magnification, A, x40; $B$, $\times 100 ; C, \times 200 ; D, \times 400 ; E$ to $G, \times 600 ; H$ to $K, \times 400$.

WT1 (Figure 1I), indicating a Müllerian origin and serous type, respectively. However, malignant cell nuclei completely lacked p53 expression (Figure 1J), indicating a truncating TP53 mutation. The lack of immunoreactivity for GATA3 (Figure $1 \mathrm{~K}$ ) excluded the possibility of a metastasis originating from the breast carcinoma.

Histopathological findings. The VATS pleural biopsy specimen revealed diffusely growing tumor cells and a lack of papillary architecture. Irregularly shaped and sized clusters of tumor cells were delineated by infiltrating borders containing obviously desmoplastic stroma (Figure 2A). The individual tumor cells exhibited both cytoplasmic and nuclear alterations and possessed relatively distinct borders with abundant eosinophilic cytoplasm. In several foci, the cytoplasm was either intensely eosinophilic or clear, with variably-sized vacuoles. The tumor cell nuclei displayed marked pleomorphism and membrane irregularity. Consistent with the cytopathological features, some tumor cell nuclei were more than 5 -fold larger than those in the adjacent tumor cells, and cells harboring the former nuclei thus resembled multinucleated giant cells (Figure 2B). These cells also had a degenerated appearance, with apparent chromatin clumping or smudging. Frequent mitotic figures and a few atypical mitoses were observed. Consistent with the cytology specimens, occasional intracytoplasmic vacuoles were detected. Coagulative tumor cell necrosis was evident in some areas of the biopsied specimen, and scattered nests or 


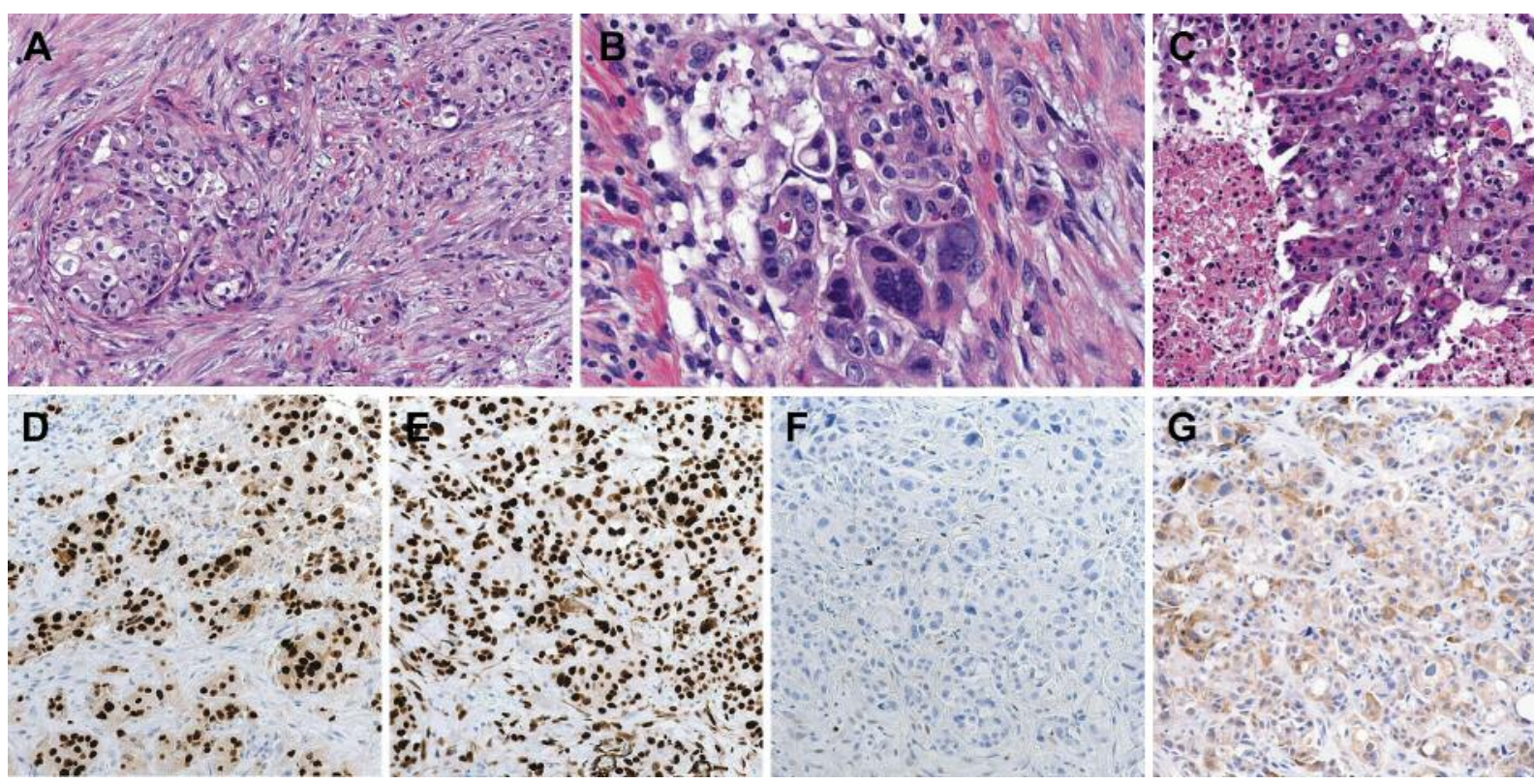

Figure 2. Histopathological findings of a pleural biopsy obtained via video-assisted thoracoscopic surgery. A: Diffusely growing tumor cells were found, devoid of papillary architecture. They were variably sized, irregularly shaped clusters of tumor cells with infiltrating borders and obvious desmoplastic stroma. B: Intensely eosinophilic or clear cytoplasm with variably sized vacuoles, markedly pleomorphic nuclei with irregular membranes was noted, and with frequent mitotic figures and a few atypical figures. C: Coagulative tumor cell necrosis and nests or clusters of surviving tumor cells were apparent. Cells showed strong, uniform nuclear paired box $8(D)$ and Wilms tumor 1 (E) expression. F: p53 Expression was completely absent, indicating TP53 mutation. G: Lack of nuclear GATA-binding protein 3 expression was noted. A to C, hematoxylin and eosin staining; $D$ to $G$, polymer method. Original magnification, $A, \times 100 ; B, \times 400 ; C, \times 100 ; D$ to $G, \times 200$.

clusters of surviving tumor cells were observed adjacent to the necrotic areas (Figure 2C). Lymphatic tumor emboli were also identified. The stroma was densely fibrotic in some areas and contained inflammatory cells and fibrinous debris. Immunostaining of the pleural tumor yielded results consistent with the immunocytochemical findings. Specifically, the tumor cells were uniformly positive for PAX8 (Figure 2E) and WT1 (Figure 2F), and exhibited a complete lack of p53 expression (Figure 2G). The surrounding inflammatory cells and reactive mesothelial cells exhibited patchy p53 expression with a variable level of staining intensity (i.e. wild-type p53 expression pattern). Again, the tumor cells were negative for GATA3 (Figure $2 \mathrm{H}$ ), as well as cytokeratin 5/6 and D2-40, which excluded the possibility of MBC and malignant mesothelioma.

\section{Discussion}

In this report, we have described our experience with a case of primary HGSC that was repeatedly misdiagnosed as pMBC in a patient who had previously been treated for primary breast carcinoma. Female breast carcinoma accounted for approximately one-fourth of all newly diagnosed carcinoma cases worldwide in 2018 (16). In the
Republic of Korea, the incidence of breast carcinoma has increased continuously since 2000 , and this malignancy is among the top five carcinomas affecting women of all ages (17). The bone is the most common site of first distant metastasis of breast carcinoma, followed by the lungs, brain, and liver (18). In contrast, MBC rarely involves the peritoneal cavity. Although only a few articles have reported the incidence of $\mathrm{pMBC}$, this disease is undoubtedly a significant cause of morbidity and mortality in patients with breast carcinoma $(19,20)$.

Lobular histology, higher histological grade, and nonluminal A subtype have been identified as significant pathological predictors of the development of MBC (21). Moreover, MBC with peritoneal involvement occurs more frequently in patients with advanced-stage primary carcinoma or a history of multiple nonperitoneal metastases (3). In our case, the patient had early-stage (pT1N0) primary breast carcinoma with a histological grade of 2 , and the tumor exhibited diffusely strong ER and PR expression (i.e. luminal A subtype). Moreover, she had no prior history of recurrence or nonperitoneal metastasis. Taken together, these findings suggested a low likelihood of pMBC.

In a patient with history of carcinoma, the primary site of a malignant effusion is often the site of a pre-existing 
malignancy. Consequently, we did not consider the possibility of a primary peritoneal HGSC during several rounds of cytological analysis. However, the features of our case demonstrate the importance of a pathological focus on the cytomorphological features, as the possibility of a second primary site of malignancy cannot be excluded completely. In such cases, a pattern recognition approach can facilitate the differential diagnosis. For example, on a liquid-based cytological slide, pMBC cells in a malignant effusion would generally present with a cannonball appearance or as acinar groups and single cells, although single linear formations of signet ring-like cells have been observed in cases involving a primary invasive lobular carcinoma of the breast. Specimens of hormone receptor-positive breast carcinoma contain tumor cells with low-to-intermediate-grade nuclear atypia and a low mitotic rate. A papillary structure with fibrovascular cores or psammomatous calcifications is rarely observed in histological primary breast carcinomas other than invasive micropapillary carcinomas. In contrast, cellular specimens of primary peritoneal HGSCs usually comprise predominantly papillary clusters with variable sizes and occasional psammoma bodies, although cannonballs or acinar groups may be observed (22). HGSC tumor cells contain large, pleomorphic nuclei with hyperchromasia and conspicuous nucleoli and exhibit markedly increased mitotic activity and atypical mitotic figures. In our case, all the cytology specimens were hypercellular and predominantly contained arborizing papillary fragments, as well as large tumor cells with severe nuclear pleomorphism, multiple prominent nucleoli, and frequent mitoses, some of which showed atypical figures.

Immunocytochemistry can help to identify the cell type and origin of a metastatic tumor. Previous studies indicate that the expression of GATA3, gross cystic disease fluid protein-15, and mammaglobin in tumor cells favors MBC (23), whereas the expression of PAX8 favors a tubo-ovarian or peritoneal origin and WT1 positivity indicates the serous subtype (24). The predictive value of these markers for distinguishing between the breast or peritoneal/ovarian origin of malignant cells has been well-demonstrated $(25,26)$. Both the lymph node and distant metastatic sites of MBCs often exhibit ER/PR/HER2 expression patterns that differ from those of the corresponding primary breast carcinoma $(27,28)$. Furthermore, similar hormone receptor expression patterns were observed in primary breast carcinoma and HGSC tissues of approximately one-third of cases in a previous study (1). In summary, although the ER/PR/HER2 immunoprofile is therapeutically important, it may not facilitate the differential diagnosis between HGSC and MBC (1).

In contrast to the cytological specimens, we did not observe the papillary structure in VATS biopsy specimens collected from our case. We additionally observed nuclear and cytoplasmic alterations that are not commonly observed in HGSC. The nuclear features included extreme enlargement with an irregular outline, a highly abnormal outline that mimicked a multinucleated giant cell, and a degenerated chromatin pattern with occasional clumping or smudging. The cytoplasm was abundant and contained intracytoplasmic vacuolations and microvacuoles, the aggregation of which caused a foamy appearance. Some areas contained intensely eosinophilic cytoplasm. The concomitant increases in the nuclear and cytoplasmic volumes resulted in a low nucleusto-cytoplasm ratio, characteristic of the histological features of surviving tumor tissues in response to chemotherapy. Our observation of the same cytomorphology in the ascitic fluid after chemotherapy further confirmed that these nuclear and cytoplasmic alterations were caused by chemotherapy. We therefore attributed the misinterpretation of the pleural lesions as $\mathrm{pMBC}$ to the absence of the typical architectural pattern of HGSC, as well as the chemotherapy-induced alterations in cellular morphology. Our observations suggest that when examining recurrent or metastatic tumors in a patient who has received postoperative chemotherapy, the pathologist should recognize the morphological changes attributable to treatment, as these may increase the challenges associated with diagnosis and determination of the histological type and grade.

In summary, previous studies have emphasized the importance of differentiating between pMBC and HGSC via peritoneal fluid cytology in a patient with a history of breast carcinoma. We hereby present an additional case in which HGSC was repeatedly misinterpreted, as we believe that our experience further underscores the need for awareness regarding the diagnostic hallmarks of HGSC, such as a dominant papillary pattern with positive immunoreactivity for PAX8 and WT1 and a lack of GATA3. Although peritoneal HGSC is rare, this awareness would reduce the likelihood of misdiagnosis and ensure accurate management, and would ultimately improve patient survival.

\section{Conflicts of Interest}

None of the Authors have any conflicts of interest to declare regarding this study.

\section{Authors' Contributions}

All Authors made substantial contributions to the conception and design of the study; the acquisition, analysis, and interpretation of the data; drafting of the article; critical revision of the article for important intellectual content; and the final approval of the version to be published.

\section{Acknowledgements}

This research was supported by a grant from the National Research Foundation of Korea (NRF), funded by the Korean government 
(MSIT) (2018R1C1B5043725). All Authors would like to thank Dr Sung-Im Do (Department of Pathology, Kangbuk Samsung Hospital, Seoul, Republic of Korea) for her valuable comments and support.

\section{References}

1 Na K, Lee JY, Sung JY, Kim GM, Koo JS and Kim HS: Comparative clinicopathological and cytomorphological analyses of peritoneal carcinomatosis associated with metastatic breast carcinoma and primary peritoneal/ovarian carcinoma in patients with a history of breast carcinoma. Virchows Arch 473: 165-175, 2018. PMID: 29926183. DOI: 10.1007/s00428-018-2390-5

2 Garg R, Zahurak ML, Trimble EL, Armstrong DK and Bristow RE: Abdominal carcinomatosis in women with a history of breast cancer. Gynecol Oncol 99: 65-70, 2005. PMID: 15979132. DOI: $10.1016 /$ j.ygyno.2005.05.013

3 Tserkezoglou A, Kontou S, Hadjieleftheriou G, Apostolikas N, Vassilomanolakis M, Sikiotis K, Salamalekis E, Tseke P and Magiakos G: Primary and metastatic ovarian cancer in patients with prior breast carcinoma. Pre-operative markers and treatment results. Anticancer Res 26: 2339-2344, 2006. PMID: 16821613.

4 Kim JY, Kim SH and Kim HS: Promoter methylation downregulates osteoprotegerin expression in ovarian carcinoma. Anticancer Res 39: 2361-2367, 2019. PMID: 31092428. DOI: 10.21873/anticanres.13353

5 Kim HG, Park MS, Sung JY, Kim YW, Kim HS and Na K: Tumor-specific expression of insulin-like growth factor II mRNA-binding protein 3 independently predicts worse survival of patients with adenocarcinoma of the ampulla of Vater. Anticancer Res 39: 4947-4955, 2019. PMID: 31519600. DOI: 10.21873/anticanres. 13683

6 Jung YY, Woo HY and Kim HS: Targeted genomic sequencing reveals novel TP53 in-frame deletion mutations leading to p53 overexpression in high-grade serous tubo-ovarian carcinoma. Anticancer Res 39: 2883-2889, 2019. PMID: 31177126. DOI: 10.21873/anticanres.13417

7 Bae GE, Yoon N, Cho EY, Kim HS and Cho SY: Clinicopathological and molecular characteristics of mammary adenoid cystic carcinoma with adipocytic differentiation with emphasis on the identification of a novel BRAF mutation. Anticancer Res 39: 369-374, 2019. PMID: 30591482. DOI: 10.21873/anticanres.13121

8 Bae GE, Do SI, Kim K, Park JH, Cho S and Kim HS: Increased sphingosine kinase 1 expression predicts distant metastasis and poor outcome in patients with colorectal cancer. Anticancer Res 39: 663670, 2019. PMID: 30711943. DOI: 10.21873/anticanres.13161

9 Park CK and Kim HS: Clinicopathological characteristics of ovarian metastasis from colorectal and pancreatobiliary carcinomas mimicking primary ovarian mucinous tumor. Anticancer Res 38: 5465-5473, 2018. PMID: 30194204. DOI: 10.21873/anticanres. 12879

10 Chung T, Do SI, Na K, Kim G, Jeong YI, Kim YW and Kim HS: Stromal p16 overexpression in gastric-type mucinous carcinoma of the uterine cervix. Anticancer Res 38: 3551-3558, 2018. PMID: 29848709. DOI: 10.21873/anticanres.12627

11 Joo JW, Kim HS, Do SI and Sung JY: Expression of zinc finger and BTB domain-containing 7A in colorectal carcinoma. Anticancer Res 38: 2787-2792, 2018. PMID: 29715100. DOI: 10.21873 /anticanres. 12522
12 Sung JY, Jung YY and Kim HS: Clinicopathological characteristics and KRAS mutation status of endometrial mucinous metaplasia and carcinoma. Anticancer Res 38: 27792786, 2018. PMID: 29715099. DOI: 10.21873/anticanres.12521

13 Jung YY, Sung JY, Kim JY and Kim HS: Down-regulation of Bcell translocation gene 1 by promoter methylation in colorectal carcinoma. Anticancer Res 38: 691-697, 2018. PMID: 29374692. DOI: 10.21873 /anticanres.12274

14 Kim HN, Woo HY, Do SI and Kim HS: Targeted sequencing of tubo-ovarian and peritoneal high-grade serous carcinoma with wild-type p53 immunostaining pattern. In Vivo 33: 1485-1492, 2019. PMID: 31471396 . DOI: 10.21873 /invivo. 11628

15 Kim M and Kim HS: Clinicopathological characteristics of welldifferentiated papillary mesothelioma of the peritoneum: A single-institutional experience of 12 cases. In Vivo 33: 633-642, 2019. PMID: 30804152. DOI: 10.21873/invivo.11521

16 Bray F, Ferlay J, Soerjomataram I, Siegel RL, Torre LA and Jemal A: Global cancer statistics 2018: Globocan estimates of incidence and mortality worldwide for 36 cancers in 185 countries. CA Cancer J Clin 68: 394-424, 2018. PMID: 30207593. DOI: $10.3322 /$ caac. 21492

17 Jung KW, Won YJ, Kong HJ, Lee ES and Community of Population-Based Regional Cancer Registries: Cancer statistics in Korea: Incidence, mortality, survival, and prevalence in 2015. Cancer Res Treat 50: 303-316, 2018. PMID: 29566481. DOI: $10.4143 /$ crt.2018.143

18 Patanaphan V, Salazar OM and Risco R: Breast cancer: Metastatic patterns and their prognosis. South Med J 81: 11091112, 1988. PMID: 3420442. DOI:

19 Tuthill M, Pell R, Guiliani R, Lim A, Gudi M, Contractor KB, Lewis JS, Coombes RC and Stebbing J: Peritoneal disease in breast cancer: A specific entity with an extremely poor prognosis. Eur J Cancer 45: 2146-2149, 2009. PMID: 19464163. DOI: $10.1016 /$ j.ejca.2009.04.027

20 Song Y, Barry WT, Seah DS, Tung NM, Garber JE and Lin NU: Patterns of recurrence and metastasis in BRCA1/BRCA2associated breast cancers. Cancer, 2019. PMID: 31581314. DOI: $10.1002 /$ cncr.32540

21 Bertozzi S, Londero AP, Cedolini C, Uzzau A, Seriau L, Bernardi S, Bacchetti S, Pasqual EM and Risaliti A: Prevalence, risk factors, and prognosis of peritoneal metastasis from breast cancer. Springerplus 4: 688, 2015. PMID: 26576331. DOI: 10.1186/s40064-015-1449-x

22 Pereira TC, Saad RS, Liu Y and Silverman JF: The diagnosis of malignancy in effusion cytology: A pattern recognition approach. Adv Anat Pathol 13: 174-184, 2006. PMID: 16858151. DOI: 10.1097/00125480-200607000-00004

23 Gown AM, Fulton RS and Kandalaft PL: Markers of metastatic carcinoma of breast origin. Histopathology 68: 86-95, 2016. PMID: 26768031. DOI: 10.1111/his.12877

24 Sheikh UN, Cohen C and Siddiqui MT: Utility of folate receptor alpha immunohistochemistry in cytology specimens of metastatic breast carcinoma, metastatic serous carcinoma of Mullerian origin, and primary lung adenocarcinoma. Diagn Cytopathol 44: 369-376, 2016. PMID: 26875861. DOI: $10.1002 / \mathrm{dc} .23448$

25 Shield PW, Papadimos DJ and Walsh MD: GATA3: A promising marker for metastatic breast carcinoma in serous effusion specimens. Cancer Cytopathol 122: 307-312, 2014. PMID: 24421220. DOI: $10.1002 /$ cncy. 21393 
26 Waters L, Crumley S, Truong L, Mody D and Coffey D: PAX2 and PAX8: Useful markers for metastatic effusions. Acta Cytol 58: 60-66, 2014. PMID: 24334997. DOI: 10.1159/000356426

27 Rossi S, Basso M, Strippoli A, Dadduzio V, Cerchiaro E, Barile R, D'Argento E, Cassano A, Schinzari G and Barone C: Hormone receptor status and HER2 expression in primary breast cancer compared with synchronous axillary metastases or recurrent metastatic disease. Clin Breast Cancer 15: 307-312, 2015. PMID: 25922284. DOI: 10.1016/j.clbc.2015.03.010

28 Nakayama Y, Nakagomi H, Omori M, Inoue M, Takahashi K, Maruyama M, Takano A, Furuya K, Amemiya K, Ishii E and
Oyama T: Benefits of using the cell block method to determine the discordance of the HR/HER2 expression in patients with metastatic breast cancer. Breast Cancer 23: 633-639, 2016. PMID: 25968348. DOI: 10.1007/s12282-015-0615-x

Received March 12, 2020

Revised April 1, 2020

Accepted April 8, 2020 